\title{
Las teorías implícitas en la práctica de la enseñanza
}

\section{The implicit theories in the practice of teaching}

Blanca de la Luz Fernández Heredia

Universidad Michoacana de San Nicolás de Hidalgo (UMSNH)

\section{Resumen}

La práctica de la enseñanza ha sido influida por múltiples factores que están presentes en la acción docente. Monereo afirma que el docente "es muchos profesores en uno", esta concepción está muy acorde con la visión del profesional flexible que se busca en la actualidad, con capacidad de adaptación a distintos grupos de estudiantes, pero sin traicionar sus concepciones y convicciones respecto a la forma en que se enseña y se aprende. Dichas representaciones son consecuencia del conocimiento semántico y prototípico de un dominio, que se construye por procedimientos asociativos y experiencias episódicas, además de constituir las teorías implícitas, que no son ideas aisladas, sino un conjunto organizado y coherente de conocimientos sobre el mundo físico y social. Por todo ello, cuando el docente piensa lo que va a hacer en clase, refleja la realidad de lo que está dentro de su estructura mental. De este modo, el vínculo entre la práctica docente y las teorías implícitas resulta más fuerte e importante de lo que se esperaría de ahí que el objetivo de este trabajo es realizar un análisis reflexivo sobre la forma en que las teorías implícitas influyen y determinan la práctica docente que realizan los profesores en el aula. Se debe considerar al profesor como un profesional reflexivo, aspecto que debe estimularse en la formación del profesorado, para conducirlo a la propuesta teórica de las teorías implícitas, además de instruirlo sobre la manera en que éstas se relacionan con la práctica de su enseñanza.

Palabras clave: teorías implícitas, práctica docente, profesor reflexivo, enseñanza, formación de profesores.

Nota del autor

Blanca de la Luz Fernández Heredia, Facultad de Psicología, Universidad Michoacana de San Nicolás de Hidalgo (UMSNH).

La correspondencia en relación con este artículo debe dirigirse a Blanca de la Luz Fernández Heredia, Facultad de Psicología, Universidad Michoacana de San Nicolás de Hidalgo. Calz. Ventura Puente 115, Cuauhtémoc, C. P. 58000, Morelia, Mich.

Dirección electrónica: bfernandezheredia@gmail.com Tel.: 4433399258 


\begin{abstract}
The practice of teaching is influenced by multiple factors that are present in the teaching action. Monereo states that a teacher is many teachers in one, this thought according to the vision of the flexible professional that is currently sought, with the ability to adapt to different groups of students, but without betraying their conceptions and convictions regarding the way that is taught and learned. Therefore, when the teacher thinks about what he is going to do in class, he reflects the reality of what is inside his mental structure. In this way, the link between the teaching practice and the implicit theories is stronger and more important than would be expected, so that the objective of this work is to carry out a reflexive analysis on the way in which the implicit theories influence and determine the teaching practice carried out by teachers in the classroom. Taking the teacher into account as a reflective professional, an aspect that should be encouraged in the training offered to teachers. To lead him to the theoretical proposal of the implicit theories and how they relate to the practice of his teaching.
\end{abstract}

Keywords: implicit theories, teaching practice, reflective teacher, teaching, teacher training.

La práctica de la enseñanza está influida por múltiples factores: desde la propia formación académica del docente, hasta las singularidades de la escuela donde éste labora. A partir de tal perspectiva, podría afirmarse que la práctica docente está determinada por una serie de factores tanto sociales, históricos, culturales, como personales, de quien enseña.

Al respecto, Monereo (2011) afirma que la acción de un docente apuesta por la cultura, el saber y los procesos de formación en él mismo $\mathrm{y}$ en sus estudiantes. Por lo que, este docente es muchos profesores en uno, alguien que es capaz de ajustar sus concepciones, estrategias y sentimientos a cada contexto educativo. Dicha posición es contraria a la identidad monolítica e inmutable que se propone en la identidad docente. En este sentido, Monereo \& Badía (2011) mencionan que "la coherencia y consistencia de los discursos y las prácticas docentes quedaría reflejada en la poca variabilidad de lo que se cree, se hace, se dice o se siente, dado que existe una única verdad a transmitir, por lo que comportarse profesionalmente implica actuar siempre de un mismo modo, explicando básicamente lo mismo, en todo tiempo y lugar" (p. 1). De este modo, en la actualidad se busca un profesional flexible, capaz de adaptarse a distintos grupos de alumnos y alumnas, pero sin traicionar sus concepciones y convicciones respecto a la forma en que se enseña y se aprende.

En trabajos realizados por MonroyFarías (1998), se encontró que el estudio del pensamiento del profesor puede ayudar a comprender mejor cómo y por qué el docente realiza la práctica de la enseñanza de una manera determinada, es decir, el conjunto de creencias, concepciones y valores que el profesor posee influye en su quehacer cotidiano. De esta forma, el pensamiento didáctico de los profesores y las 
profesoras manifiesta teorías, creencias y valores que pueden nutrirse con principios teóricos cuyo fin es una práctica docente determinada.

En este sentido, Reyes y Perafán (2001) mostraron que el pensamiento práctico de los docentes es más que una adición de ideas, creencias y constructos. Es un impulso, un poder dentro del aula de clase que se convierte en una limitante cuando el pensamiento no evoluciona de acuerdo con las necesidades circunstanciales.

Por otra parte, Marrero (1993) señala que en muchas ocasiones se encuentra que los cursos de formación diseñados para los docentes no causan los efectos esperados por las autoridades educativas, debido a que es difícil cambiar las creencias, ideas y constructos de los docentes, muchas veces edificadas en años no solo de servicio profesional, sino también de formación en las aulas por diferentes tipos de profesores. Por todo esto, parece conveniente conocer en qué forma se van construyendo estas creencias implícitas que los docentes poseen y de qué manera determinan su práctica docente.

De igual manera, es conveniente analizar cómo es que se planea la formación del profesorado, cómo se relaciona con la reflexión que se genera en los docentes a partir de su práctica cotidiana y por qué no se perciben los resultados esperados en estos programas de formación.

Algunas de las alternativas de respuesta que surgen de manera inmediata ante este tipo de reflexiones es que en la programación de los cursos de formación docente no se parte de un diagnóstico donde el profesorado manifieste sus propias necesidades, y que tales requerimientos estén ligados a la forma cómo los profesores y profesoras conciben la enseñanza y el aprendizaje. Estos analizan simultáneamente y de manera clara, cómo impactan en la práctica de la enseñanza los resultados de la evaluación docente que se realizan en las instituciones escolares. Por otra parte, determinar en forma clara si estos docentes reflexionan sobre los resultados que obtuvieron en su evaluación docente, se podría determinar en gran medida qué tipo de cursos de formación docente son los más convenientes para fortalecer o desarrollar las habilidades en la enseñanza.

\section{El profesor como profesional reflexivo}

La necesidad de partir de una base reflexiva del quehacer docente, con el objetivo de entender la forma cómo se abordan las situaciones problemáticas de dicha práctica, llevaría al planteamiento de recuperar -como elemento legítimo y necesario-, el proceso de la reflexión sobre la acción en la práctica de la enseñanza.

A partir de las aportaciones de Donald Shön (1983; 1992) sobre los profesionales reflexivos, se inició un análisis de cómo realmente los profesionales de cualquier área realizan su labor. Para ello, se han de reconocer dos fases: conocimiento en la acción y reflexión en la acción.

Desde esta perspectiva, la práctica docente se construye diariamente en un conocimiento tácito e implícito sobre el cual no se ejerce un control específico. Existen acciones que 
se realizan de manera espontánea sin que se tenga que pensar para ejecutarlas. Estas son comprensiones de las cosas interiorizadas, de tal forma que resultaría difícil dar una explicación o describir el conocimiento que revelan. En la mayoría de las ocasiones no se hace conciencia de haber aprendido tales acciones, simplemente se descubren al realizarlas.

En este tipo de situaciones, el conocimiento no precede a la acción, sino que está en la acción. Según Shön (1992), no existen evidencias que señalen que un "saber cómo" esté constituido como un conjunto de reglas estructuradas que anteceden a la acción y las cuales funcionan como aplicación de decisiones. Para estos contextos, el conocimiento no se aplica a la acción, sino que se encuentra de manera tácita dentro de la propia acción, por ende, se considera un conocimiento en la acción.

La segunda fase se reconoce después de un evento que queda fuera de lo sucedido habitualmente y obliga a pensar en lo que se realiza. De tal forma que genera una reflexión de la acción, incluso mientras se está haciendo. Con ello se permite realizar un análisis en relación con la situación $\mathrm{y}$, en consecuencia, ésta se reconduce.

En tales situaciones, la reflexión tiende a focalizarse interactivamente sobre los resultados de la acción, sobre la acción en sí misma y sobre el conocimiento intuitivo implícito en la acción. Para considerar al profesor como un profesional y un especialista en la enseñanza, sometido de manera cotidiana a situaciones que son habituales y le permiten desarrollar un repertorio de expectativas, imágenes y técnicas base para sus decisiones, se aprende, por tanto, a buscar y encontrar alternativas sobre cómo responder a lo que encuentra. Esta experiencia es la que alimenta día con día su práctica.

Conforme pasan los años en servicio, los docentes desarrollan una práctica estable y repetitiva, lo cual les permite adoptar un conocimiento sobrentendido y espontáneo. Esta situación lo lleva a confiarse y dar por hecho que ya está especializado. Sin embargo, en la medida que existen casos ajenos a ese control y a su experiencia, situaciones que parecen incompatibles y conflictivas, el docente debe reflexionar sobre las posibles soluciones que no había encontrado antes, por la falta de necesidad. Por ello, deberá entender y solucionar el nuevo caso cuyas características serán novedosas y singulares.

Esta reflexión confronta su conocimiento práctico para la situación que escapa de su control o para aquella donde las respuestas conocidas no se expresan de manera satisfactoria. Entonces, el docente busca generar una nueva propuesta de solución reflexionando con ello en la acción, respuesta que puede dar incluso en el momento mismo del evento. En estas ocasiones es cuando se logra una mejor evidencia de la movilización de recursos profesionales que no se pueden explicar - debido a que no son observables -, en tanto el mismo docente no los manifieste. Para lograr un cambio es necesario crear nuevos enfoques, nuevas formas de entender los 
problemas que no se les habían presentado con anterioridad, lo que para Shön (1983) convierte al docente en "un investigador en el contexto de la práctica" (p.56).

Por otra parte, cuando el fenómeno que se aborda soslaya las condiciones ordinarias del conocimiento en la práctica (mostrándose como único o inestable), el profesional puede hacer emerger y criticar su visión inicial del fenómeno, construir una nueva descripción del mismo y probarla mediante un experimento sobre la marcha. En ocasiones, llega a una nueva teoría del fenómeno al articular un presentimiento que tenía sobre él.

\section{La formación del profesorado}

Cuando se habla de cambio en los procesos educativos, ya sea de una región, un pueblo o un país, las miradas se centran invariablemente en la figura del docente y en su formación para que dicho proceso se lleve a cabo.

En los últimos diez años, ésta ha sido una factura, en muchos casos, difícil de pagar, debido al poco respeto que la figura docente ha venido manifestando, de ahí que pocos se queden desempeñando esta profesión, donde, para hacerlo, persiste la deteriorada situación a nivel mundial en la cuestión económica y laboral (Marcelo, 1999). Sin embargo, para los que se quedan y desempeñan esta actividad, la reflexión sobre el carácter social tiene muy poco peso argumentativo para que el docente dé el paso hacía la profesionalización de su práctica (Zarzar-Charur, 2001).
Delval (2004) señala que la preparación de los docentes debería cambiar mucho en cuanto a los objetivos de la enseñanza para encaminarla más a resolver las necesidades de los alumnos, que al sometimiento de los mismos, $\mathrm{y}$, adecuarse, por supuesto, a los nuevos modelos culturales y sociales.

La escuela en la actualidad resulta algo importante, pero el interés por ella no es nuevo ni tampoco resulta casual. Su importancia se explica por el papel trascendental que desempeña en la educación de las nuevas generaciones, por las insatisfacciones que con frecuencia sus resultados provocan y por la necesidad de transformación de la que la propia escuela está urgida ante los cambios que las actuales condiciones del desarrollo científico y social demandan. En la actualidad, el creciente interés por la escuela también se explica porque de ella se espera que sirva de motor para promover el bienestar de cada sociedad, que consiga la formación de ciudadanos con capacidad para compartir con éxito en el panorama nacional e internacional y que los prepare para la superación de los desafíos futuros, para contribuir eficazmente al desarrollo personal y a la integración social (Castaño \& Fernández, 2014). Sin embargo su función debe centrarse en formar personas con capacidad crítica y de reflexión, antes que concentrarse en ella misma como organismo administrativo y burocrático. Hoy en día, la gran información que los alumnos reciben por otros medios debería analizarse en la escuela, ya que tanto la televisión como el 
internet no enseñan a pensar por sí mismos, $y$ menos aún a criticar la realidad de lo que ahí se informa, de esta manera la función de la escuela resulta muy poco eficaz para ambas actividades.

Desde esta perspectiva, la formación docente debe de centrarse en la necesidad actual de los alumnos. Los docentes tienden a enseñar cómo fueron enseñados, dejan la falsa idea de que ser docente es únicamente dominar la disciplina y los contenidos, carece de valor la motivación, el gusto y el ánimo que se imprima en un aula a la actividad docente (Fernández, 1996).

Ante tal realidad, los nuevos centros de formación de docentes deberían ser más cercanos a los nuevos paradigmas de enseñanza: enseñar al docente que la escuela es un laboratorio donde se aprende a reflexionar sobre los problemas y situaciones reales, y que descubrir es la forma en que el alumno adquiere la habilidad para pensar por sí mismo, convirtiéndose así en un ser autónomo, con procesos metacognitivos $\mathrm{y}$ autorregulatorios que favorezcan en el aprendizaje significativo.

De acuerdo con un estudio comparativo realizado entre cuatro países (Argentina, Brasil, Perú y Uruguay), se sabe que para los programas de formación docente no existen dispositivos institucionales que favorezcan la producción, sistematización, valoración y difusión de este conocimiento básico en el cuerpo docente, ya que, por lo general, prevalece el abordaje de contenidos "teóricos" que se alejan de la realidad vivida día a día en los centros de trabajo y en la sociedad en general (Tenti, 2007).
Esta situación que se vive diariamente en las aulas lleva a los alumnos a considerar que dominar el arte de la memoria es uno de los métodos infalibles para ser exitoso en la escuela e ignorar que ello no beneficia en nada las habilidades para una construcción de aprendizaje significativo, que les permitan movilizar $y$ aplicar esos saberes en cualquier contexto. Sin embargo, tanto docentes como alumnos llevan implícitas teorías sobre la enseñanza, los primeros, y del aprendizaje, los segundos.

\section{Las teorías implícitas}

El pensamiento o cognición del profesorado se define en forma genérica como el conjunto de reflexiones que realiza el profesor en formación o en servicio, fundamentado en sus creencias y conocimientos sobre la enseñanza, los estudiantes y el contenido, resume todo en la propuesta de estrategias de resolución de problemas sustanciales a la enseñanza del aula. Marrero (1988) lo define como conocimiento experto, profesional o laboral de los docentes.

Desde distintos ángulos, se considera que los docentes desempeñan su profesión con un conjunto de conocimientos, habilidades $\mathrm{y}$ creencias que requieren para afrontar las complejas situaciones cotidianas que se presentan en el aula. Dichas concepciones guían la práctica docente de forma implícita o explícita. Estos múltiples ángulos o perspectivas fueron reunidos por Marrero (1988) en cuatro conocimientos principales: 1) lo científico; 2) la creencia; 3) el contenido; y 4) la práctica como conocimiento. 
Lo científico como conocimiento retoma la Teoría de los Constructos de George Kelly (1963). En ésta se intenta determinar qué tipos de constructos emplean los docentes para discriminar tareas concretas, debido a que se sostiene que el pensamiento de los docentes se constituye por una serie de constructos que le permite explicar, interpretar, ordenar, prever y tomar decisiones (Jiménez-Llanos, 2002).

Por su parte, la creencia como conocimiento se fundamenta en la Teoría de Esquemas de Hiztman, según la cual los profesores disponen de esquemas pertenecientes a situaciones didácticas (conceptos prototípicos) que les permiten resolver situaciones al interior del aula, que van desde un bloqueo en el aprendizaje hasta respuestas rutinarias y con el paso de los años en servicio, les permitirán aumentar sus redes semánticas, cambiar conceptos y mejorar o producir nuevos conceptos.

Los contenidos de las asignaturas cobran particular importancia y se sustentan en los saberes que un docente domina acerca de determinados ámbitos de la realidad, aunque, definitivamente, tal conocimiento incide en la forma cómo piensan y organizan los docentes su práctica. Para Montero (2001), indagar en el conocimiento que los profesores tienen de sus materias y en cómo lo trasladan al aula, es un asunto fundamental para los investigadores, lo que Shulman (1987) denominó paradigma perdido debido a la escasa atención que recibía el papel del contenido en la formación docente.
En la actualidad, las investigaciones sobre el profesor y su pensamiento destacan el carácter eminentemente práctico que éste posee. Así, los términos conocimiento artesanal, conocimiento práctico, conocimiento profesional, pensamiento reflexivo, dilemas, etc., han llegado a dominar las investigaciones en el campo de la enseñanza. Por lo anterior, ahora se concibe al profesor como profesional capaz de tomar decisiones en situaciones complejas y reflexionar sobre su quehacer para evaluar y ajustar sus acciones.

Para llevar a cabo esta reflexión de su práctica, emplea una serie de elementos entre los que intervienen lo individual, lo social, el contexto y la normativa. Sin embargo, no sería correcto soslayar, en este proceso dialéctico, su formación a partir de un cuerpo de representaciones sobre un área del conocimiento, determinando las concepciones que cada sujeto activa en función de las demandas de la tarea a resolver y proporcionando las características de los modelos mentales movilizados en cada uno de los diferentes contextos.

Las representaciones que cada individuo tiene están formadas por conocimiento semántico, esquemático, prototípico, relativo a un dominio y se construye por procedimientos asociativos a partir de experiencias episódicas; a este conjunto se le conoce actualmente como teorías implícitas. De acuerdo a Rodrigo (1997), estas teorías son las que proporcionan el marco conceptual, epistemológico y ontológico a partir del cual el sujeto elabora las teorías de dominio, 
de manera que las primeras restringen tanto la selección de la información procesada como las relaciones establecidas entre los elementos de esa información.

Se opta por llamarlas teorías porque raramente se constituyen en ideas aisladas, se identifican como un conjunto más o menos organizado y coherente de conocimientos sobre el mundo físico o social. El término "implícito" se refiere a la carencia de accesibilidad a la conciencia, lo cual les confiere una apariencia de realidad que elimina toda sensación de provisionalidad en las visiones del hombre de la calle sobre el mundo (Rodrigo, 1993).

En el ámbito del pensamiento del docente, se definen las teorías implícitas del profesorado como teorías pedagógicas personales, reconstruidas sobre la base de conocimientos pedagógicos, históricamente elaborados $\mathrm{y}$ transmitidos a través de la formación y en la práctica (Marrero, 1993).

Ante esto, Fernández (2009) menciona que los profesores no aplican en el aula los métodos que les han predicado, sino los métodos que les han aplicado durante su periodo de formación, ocasionando con ello el inicio de un ciclo que parece no concluir, ya que los cursos de formación a los que se les somete, no logran generar un cambio. Nuevamente, se aprende de la práctica que se tiene en la formación y no del material que se les proporciona. Pareciera que quedan vacunados contra la posibilidad de un verdadero cambio.
De acuerdo a Coll (1987), cuando los profesores se plantean la necesidad de enseñar determinados contenidos ponen en funcionamiento, casi sin pretenderlo, una serie de ideas sobre el proceso de aprendizaje y sobre cómo se puede ayudar al alumno en este proceso.

Tal fenómeno se encuentra influenciado por los años de servicio en la docencia y la reflexión de la actividad en sí. Al ser parte de la teoría personal, se emplea como referente para la toma de decisiones sobre qué, cuándo y cómo enseñar o evaluar.

De igual manera se responde al compromiso de la misión y la visión de la institución en la que se desarrolla la docencia, y se recurre a los mismos referentes con la finalidad de desarrollar los contenidos, en función de los objetivos que persigue la institución.

\section{Las teorías implícitas en la práctica de la enseñanza}

En los últimos diez años se ha observado la práctica de los docentes con otras perspectivas (Kemmis, 2008; Zabala, 2008; Díaz-Barriga \& Hernández, 2002), al incluir en el análisis no sólo el comportamiento de lo que un docente realiza en un aula, sino que, además, se ha buscado explicar qué tipos de pensamientos motivan el quehacer de los profesores e indagar por qué piensan lo que piensan cuando están enseñando (Monereo, 2009). La diferencia entre un docente $\mathrm{y}$ otro se ha cuestionado desde la igualdad de las políticas educativas, el plan de estudios, los programas y la literatura propuesta, así como el 
manejo que se le da al espacio áulico y al abordaje de los temas. Todo esto se ha explicado a través de las concepciones y conductas asociadas a cada individuo. Éstas evolucionan mediante un proceso, que se realiza de forma más o menos consciente de reestructuración y construcción de significados, basado en la interacción y el contraste de otras ideas y experiencias situadas dentro de la zona de desarrollo potencial de cada sujeto (Bowen, 2008).

Por lo anterior, se puede decir que cuando el docente piensa lo que va a hacer en clase refleja la realidad de lo que está dentro de su estructura mental a través de la adquisición de conocimientos. Para poder manifestar lo que está dentro de su mente primeramente ha de pensar sobre su propio pensamiento, esto requiere la organización de las ideas previas que están en su estructura cognitiva.

Autores como Wittrock (1990) y MonroyFarías (1998), coinciden en señalar que el pensamiento del profesor se define como el conjunto de procesos lógicos acerca de la enseñanza que vincula las teorías implícitas y la práctica pedagógica, es decir, el pensamiento del pedagogo permite el análisis del trabajo docente. De esta manera, la forma de abordar los objetivos, las actividades y la evaluación es parte fundamental de una práctica docente, que es desarrollada a partir de la planeación que realiza el profesor en función de su experiencia y con base en las creencias que posee, tanto en la enseñanza como en el aprendizaje.
Tales creencias o representaciones, según Pozo y otros autores (Pozo \& Monereo, 2005; Pozo et al., 2009), se entienden como un conjunto de restricciones en el procesamiento de la información que determina tanto la forma de procesar esa información, como la manera de establecer relaciones desde ese procesamiento. Estas representaciones fueron adquiridas a través de procesos de aprendizaje implícito, resultado de la experiencia personal en condiciones de aprendizaje que resulta difícil comunicar y compartir, porque de forma muy probable vienen simbolizadas a partir de códigos no formales.

Las representaciones se consideran implícitas porque en la mayoría de los casos no es algo que el docente realice de forma explícita o pueda verbalizar, incluso en ocasiones ni siquiera son reconocidas por quien las pone en práctica, ya que toman en cuenta que son un saber hacer. Las representaciones implícitas están profundamente arraigadas, constituyen ideas equivocadas dado que se rigen por un realismo ingenuo. Este tipo de lógica hace que las concepciones constructivistas del aprendizaje y la enseñanza resulten profundamente contrarias a lo que intuitivamente conoce el docente y por lo tanto, difíciles de asumir.

En este sentido, se percibe el aprendizaje como una copia fiel de la realidad, al ser ésta una copia de conductas o resultados, sin que se reconozca ningún proceso de mediación psicológica. Bajo dicha percepción, la simple 
exposición al contenido y objeto del aprendizaje garantiza el resultado, concebido como una reproducción fiel de la información presentada.

A través de múltiples estudios realizados sobre las teorías implícitas con respecto al aprendizaje (Rodrigo, 1993; Fernández, Tuset, Pérez \& Leyva, 2009; Pozo \& Monereo, 2005), se ha observado que no es posible definir una sola, sino que las teorías se ajustan al escenario y las exigencias de éste. Los profesores adaptan esas teorías de acuerdo con las demandas situacionales de la tarea o actividad a desarrollar.

Esto exige asumir un pluralismo representacional dentro de un mismo sujeto.

Para hacer frente a este elemento que obstaculiza el cambio de las prácticas educativas de los docentes, distintos autores (Pozo \& Monereo, 2005; Pozo, 2002; Zelaya \& Campanario, 2001) han señalado la importancia de estudiar las concepciones de los profesores sobre la enseñanza y el aprendizaje, además de sus relaciones con la práctica docente como un aspecto clave para el cambio de estas prácticas. Para Pecharromán \& Pozo (2006), las concepciones y la práctica son dos aspectos indisociables del proceso de enseñanza, de tal manera que para lograr un cambio en las prácticas escolares. Por lo que se requiere modificar también las representaciones que tienen los profesores sobre lo que está pasando en las aulas.

\section{A manera de conclusión}

El vínculo entre la práctica docente y las teorías implícitas ha resultado más fuerte e importante de lo que se hubiera pensado con anterioridad. Los últimos años han evidenciado una relación fundamental entre lo que los docentes creen y lo que realizan como parte de su práctica diaria. Estudios específicos sobre este tema (Rodrigo, 1993; Marrero, 1993; Pérez Echeverría, Scheuer, Mateos \& Martín, 2006) han destacado que la práctica docente se encuentra fuertemente influenciada por las teorías implícitas de los profesores.

Pajares (1992), señala que durante las décadas de los años ochenta y noventa del siglo pasado, pocos investigadores se hubieran atrevido a afirmar que las creencias de los docentes podrían influir en sus percepciones y derechos, lo que influye a su vez en su desempeño en el aula, más aún, a reconocer que estas creencias permitirían de manera eficaz mejorar la práctica y la preparación de la enseñanza.

La queja de muchos investigadores es que se le ha dado poca importancia a este tema, no sólo en su campo, sino sobre todo de parte de los docentes. A este respecto, Pajares (1992) considera importante que se retomen las creencias desde la propia Teoría Social Cognitiva, debido a que conceptos tales como autoconcepto y autoestima se consideran fundamentales para las teorías fenomenológicas y humanísticas, entonces ¿por qué no explorar estas creencias como base para favorecer la formación docente?

A partir de estas premisas, la enseñanza y el desempeño de los docentes se han visto influenciados a lo largo de los años por las 
teorías que cada profesor tiene implícitas y que evidencia cuando realiza su práctica.

Al tomar como base que se ha encontrado (Marrero, 1988; Pajares, 1992) y fundamentado de manera amplia que las teorías implícitas de todas las personas se forman como parte de las experiencias de un individuo (Gómez, 2008; López-Vargas \& Basto-Torrado, 2010), además, que éstas se movilizan para la vida cotidiana y en determinados contextos, al permitir realizar predicciones de sucesos futuros y generando rutinas operativas sobre un modo correcto de actuar (Pozo \& Col, 2009). Al mismo tiempo, posibilitan a las personas predecir, interpretar y actuar en diversas situaciones, es de suma importancia conocerlas para poder explicar el actuar de los docentes en el aula.

Enestesentido, sepuede afirmar que la práctica docente tiene una gran carga de pensamientos, teorías y creencias de esa persona que realiza su práctica de enseñanza como profesor o profesora y que ésta se encuentra permeada por todo un marco referencial personal. Por ello, la planeación de las actividades, el proceso durante la clase y la evaluación del aprendizaje se desarrolla bajo el contexto de las teorías implícitas que posee quien enseña, influye en la praxis que realiza en su salón de clase.

\section{Referencias}

Bowen, J. (2008). Teorías de la Educación. Australia: LIMUSA.

Castaño, M. S., \& Fernández, A. (2014). La escuela ante la amenaza de los factores de riesgo. Revista de la Escuela de Ciencias de la Educación, 10(9), 183-196.

Coll, C. (1987). Psicología y Currículum. Barcelona: Paidós.

Delval, J. (2004). Los fines de la educación. España: Siglo XXI.

Díaz-Barriga, F., \& Hernández, G. (2002). Estrategias docentes para un aprendizaje significativo. México: McGraw Hill.

Fernández, M. (2009). La profesionalización del docente. México: Siglo XXI.

Fernández, J. (1996). Qué piensan los profesores acerca de cómo se debe enseñar. Investigación y experiencias didácticas, 14(3), 331-342. Recuperado de file://C:/Users/FPSI-LDEISY/Downloads/56871-93712-1-PB.pdf.

Fernández, M. T., Tuset, A., Pérez, R. E., \& Leyva, A. C. (2009). Concepciones de los maestros sobre la enseñanza y el aprendizaje y sus prácticas educativas en clases de ciencias naturales. Revista Enseñanza de las Ciencias, 27(2), 287-298. Recuperado de https://ddd.uab.cat/pub/edlc/02124521v27n2 /02124521v27n2p287.pdf.

Gómez, L. F. (2008). Las teorías implícitas de los profesores y sus acciones en el aula. Revista Electrónica Sinéctica, (30), 1-14. Recuperado de http://www.redalyc.org/ articulo.oa? $\mathrm{id}=99819167005$.

Jiménez-Llanos, A. B. (2002). Nivel educativo $y$ teorías implícitas de los profesores: estructura de creencias de los docentes de educación primaria, secundaria y superior 
(Tesis doctoral inédita). Universidad de la Laguna, España.

Kemmis, S. (2008). El currículum: más allá de la teoría de la reproducción. Madrid: Morata. Kelly, G. A. (1963). A theory of personality The psychology of personal constructs. [Una teoría de la personalidad - La psicología de constructos personales]. New York: W. W. Norton.

López-Vargas, B., \& Basto-Torrado, S. (2010). Desde las teorías implícitas a la docencia como práctica reflexiva. Revista Educación y Educadores, 13(2), 275-291.

Marcelo, C. (1999). Formación del profesorado para el cambio educativo. Barcelona: EUB.

Marrero, J. (1988). Teorías implícitas y planificación de la enseñanza. España: Universidad de Sevilla.

Marrero, J. (1993). Las teorías implícitas del profesorado: Vínculo entre la cultura y la práctica de la enseñanza. Madrid: Aprendizaje Visor.

Monereo, C. (2009). La evaluación auténtica en enseñanza secundaria y universitaria: Investigación e innovación. En C. Monereo, La autenticidad de la evaluación (pp. 15-28). Barcelona: Edebé.

Monereo, C. (2011). La identidad en psicología de la educación. Madrid: Narcea.

Monroy-Farías, M. (1998). El pensamiento didáctico del profesor: un estudio con profesores deficiencias histórico sociales del colegio de bachilleres y del colegio deficiencias y humanidades. Revista en
Línea. Recuperado de http://www.reduc.cl/ raes.nsf/0/041da27d1d11a95f04256baa0072 $129 \mathrm{c}$ ? O penDocument.

Montero, L. (2001). La construcción del conocimiento profesional docente. Buenos Aires: Homo Sapiens.

Pajares, F. (1992). Teachers' Beliefs and Educational Research: Cleaning Up a Messy Construct. [Creencias de los profesores y la investigacion educativa: limpiando un constructo confuso]. Review of Educational Research, 62(3), 307-332. Recuperado de http://rer.sagepub.com/content/62/3/307.full. pdf + html.

Pecharromán, I., \& Pozo, J. I. (2006). ¿Cómo sé que esverdad?: epistemologías intuitivas delos estudiantes sobre el conocimiento científico. Investigaçoes em ensino de ciencias, 11(2), 153-187. Recuperado de https://repositorio. uam.es/handle/10486/666209.

Pérez-Echeverría, M. D. P., Mateos, M., Scheuer, N., \& Martín, E. (2006). Enfoques en el estudio de las concepciones sobre el aprendizaje y la enseñanza. En N, Scheuer., J. I, Pozo., M. D. P, Pérez-Echeverría., M. D. M, Mateos., E, Martín Ortega \& M, De la Cruz. (Coords.), Nuevas formas de pensar la enseñanza y el aprendizaje. Las concepciones de profesores y alumnos (pp. 55-89). Barcelona: Graó.

Pozo, J. I. (2002). La adquisición del conocimiento científico como un proceso de cambio representacional. Investigações em ensino de ciencias, 7(3), 245-290. 
Recuperado de https://repositorio.uam.es/ handle/10486/666207.

Pozo, J. I., \& Monereo, C. (2005). La práctica del asesoramiento educativo a examen. Barcelona: Graó.

Pozo, J. I., Scheuer, N., Pérez-Echeverría, M., Mateos, M., Martín, E., \& De la Cruz, M. (2009). Nuevas formas de pensar la enseñanza y el aprendizaje (las concepciones de profesores y alumnos). Barcelona: Graó.

Reyes-Herrera, L., \& Perafán-Echeverri. (2001). Explorando creencias acerca de la naturaleza de la enseñanza en maestros deficiencias naturales del grado sexto en Bogotá, DC. Revista de la Facultad de Ciencia y Tecnología, 10, 22-33. doi: http://dx.doi.org/10.17227/ted.num105608.

Rodrigo, M. J. (1993). Las teorías implícitas: una aproximación al conocimiento cotidiano. España: Aprendizaje Visor.

Rodrigo, M. J. (1997). Del escenario sociocultural al constructivismo episódico: un viaje al conocimiento escolar de la mano de las teorías implícitas. En M. J. Rodrigo \& J. A. Puerta (Coords), La construcción del conocimiento escolar (pp.177-191). Madrid: Paidós.

Shön, D. (1983). The reflective practitioner. How profesionals think in action. [El practicante reflexivo. Como piensan los profesionales en accion.] Londres: Temple Smith.

Shön, D. (1992). La formación de profesionales reflexivos. Hacia un nuevo diseño de la enseñanza y el aprendizaje en las profesiones. Barcelona: Paidós.

Shulman, L. (1987). Knowledge and teaching: Foundations of the new reform. [Conocimiento y enseñanza: fundamentos de la nueva reforma.] Harvard educational review, 57(1), 1-23. Recuperado de http:// hepgjournals.org/doi/abs/10.17763/ haer.57.1.j463w79r56455411.

Tenti, E. (2007). La condición docente: Análisis comparado de la Argentina, Brasil, Perú y Uruguay. Buenos Aires: Siglo XXI.

Wittrock, M. (1990). La investigación de la enseñanza, III. Profesores y alumnos. Nueva York: MacMillan.

Zabala, A. (2008). La práctica educativa: Cómo enseñar. Barcelona: Graó.

Zarzar-Charur, C, (2001). La didáctica grupal. México, DF: Progreso.

Zelaya-Blandón, V., \& Campanario, J. M. (2001). Concepciones de los profesores nicaragüenses de Física en el nivel de secundaria sobre la ciencia, su enseñanza y su aprendizaje. Revista Electrónica Interuniversitaria de Formación del Profesorado, 4(1). Recuperado de http:// www.aufop.com/aufop/uploaded_files/ articulos/1224629548.pdf.

Recibido: 06/07/2017

Revisado: 13/12/2017

Aceptado: 30/04/2018 